\title{
Differential Temperature Sensitivity of Flowering Time and Crop Quality Parameters of 20 Seed-propagated Petunia Cultivars
}

\author{
Ryan M. Warner \\ Department of Horticulture, Michigan State University, East Lansing, MI \\ 48824
}

Additional index words. bedding plant, crop modeling, floriculture, plant development

\begin{abstract}
Twenty petunia (Petunia $\times$ hybrida) cultivars were grown at 14,17 , and $20^{\circ} \mathrm{C}$ to quantify the impact of temperature on time to flowering, flowering and development rates, and crop quality parameters. Increasing temperature increased vegetative development rates and reduced time to flower (TTF) for all cultivars. Linear functions generated to describe the effects of temperature on the flowering rate (1/TTF) revealed considerable variability in the temperature sensitivity of flowering rates across cultivars. The minimum temperature for the rate of progress toward flowering $\left(T_{\text {base }}\right)$ ranged from $0.15{ }^{\circ} \mathrm{C}$ for 'Damask Purple' to $7.1^{\circ} \mathrm{C}$ for 'Wave Purple'. The crop quality parameters plant height and branch and flower bud number were all influenced by interactions between cultivar and temperature. Plant height at flowering was unaffected by temperature for 13 of the 20 cultivars, whereas the height of five cultivars was lower at $20^{\circ} \mathrm{C}$ compared with $14^{\circ} \mathrm{C}$, and two cultivars were shortest at $17^{\circ} \mathrm{C}$. The branch number of six cultivars was lower at $14^{\circ} \mathrm{C}$ than at 17 or $20^{\circ} \mathrm{C}$, whereas three cultivars produced more branches at $17^{\circ} \mathrm{C}$ compared with $20{ }^{\circ} \mathrm{C}$. The branch number of 11 cultivars was not impacted by temperature. For 11 of the 20 cultivars, the flower bud number was greater at $14{ }^{\circ} \mathrm{C}$ than at $20^{\circ} \mathrm{C}$, whereas temperature did not influence the flower bud number for the other nine cultivars. The results of this work could help to improve production efficiency by allowing cultivars to be placed in temperature-response groups based on the temperature sensitivity of flowering time and/or crop quality parameters.
\end{abstract}

The rate of progress toward a developmental event, such as vegetative node appearance (development rate; the inverse of plastochron) or flowering, is primarily a function of the average daily temperature (Kanellos and Pearson, 2000; Niu et al., 2000; White and Warrington, 1984). The development rate and rate of progress toward flowering (flowering rate; the reciprocal of time to flower) generally increase as temperature increases between the base temperature $\left(T_{\text {base }}\right)$, where the rate is zero, and the optimum temperature $\left(\mathrm{T}_{\mathrm{opt}}\right)$, where the rate is maximal (Adams et al., 1997; Karlsson et al.,

Received for publication 2 Oct. 2019. Accepted for publication 17 Dec. 2019.

Published online 6 February 2020.

I gratefully acknowledge the assistance of technicians Nate DuRussel and Sue Hammar and the financial support from the American Floral Endowment, the USDA Floriculture and Nursery Research Initiative, growers supporting Michigan State University floriculture research, Michigan AgBioResearch, and USDA National Institute of Food and Agriculture Hatch project number MICL02451.

R.M.W. is an Associate Professor.

R.M.W. is the corresponding author. E-mail: warnerry@msu.edu.

This is an open access article distributed under the CC BY-NC-ND license (https://creativecommons. org/licenses/by-nc-nd/4.0/). and 'Wave Purple' $\mathrm{T}_{\text {base }}$ estimated at $2.8^{\circ} \mathrm{C}$ and $5.5^{\circ} \mathrm{C}$, respectively. In a separate experiment modeling the flowering rate in response to the average daily temperature and photosynthetic daily light integral, $\mathrm{T}_{\text {base }}$ was calculated as $7.3{ }^{\circ} \mathrm{C}$ and $5.5^{\circ} \mathrm{C}$ for petunia 'Easy Wave Coral Reef' and 'Wave Purple', respectively (Blanchard et al., 2011a).

In addition to influencing crop timing, temperature also influences crop quality parameters, such as flower production, branching, and plant height (Niu et al., 2001; Vaid et al., 2014; Warner and Erwin, 2005, 2006). In a study evaluating the influence of temperature on crop quality parameters of 14 annual bedding plant species, Vaid et al. (2014) reported increasing the temperature from 14 to $20{ }^{\circ} \mathrm{C}$ decreased flower bud or inflorescence number for 11 species; however, this increased flower bud number for torenia (Torenia fournieri) 'Clown Blue', New Guinea impatiens (Impatiens hawkeri) 'Divine Cherry Red', and gomphrena (Gomphrena globosa) 'Globe Purple'. Further increasing the temperature from 20 to $26{ }^{\circ} \mathrm{C}$ decreased flower bud or inflorescence number for all species. The influence of temperature on branching was more variable. The branch numbers of four species decreased as temperature increased, whereas 10 species showed no consistent effects of temperature on branching, and torenia 'Clown Blue' branch number increased as the temperature increased from 14 to $26{ }^{\circ} \mathrm{C}$ (Vaid et al., 2014).

The temperature sensitivity of flowering time in petunia has been evaluated for a small number of cultivars (Adams et al., 1998, 1999; Blanchard and Runkle, 2011; Blanchard et al., 2011a; Mattson and Erwin, 2003) and Petunia spp. (Guo et al., 2017; Warner, 2010; Warner and Walworth, 2010). The objectives of this work were to evaluate a larger panel of petunia cultivars to gain a better understanding of the relationships between temperature and crop timing and quality parameters and to increase our understanding of the variability of these responses within the commercial petunia germplasm pool.

\section{Materials and Methods}

for crops which have a relatively narrow marketable time window.

There is considerable variation in the temperature sensitivity of flowering time across species. For example, Blanchard and Runkle (2011) modeled the impact of temperature on the flowering rate of 17 annual bedding plant species. They identified wide variations in the thermal sensitivity (i.e., the increase in flowering rate per $1{ }^{\circ} \mathrm{C}$ increase in temperature between $\mathrm{T}_{\text {base }}$ and $\mathrm{T}_{\text {opt }}$ ) of the flowering rate both across species and among the two petunia cultivars evaluated ('Dreams Neon Rose' and 'Wave Purple'). The $\mathrm{T}_{\text {base }}$ ranged from $1.1{ }^{\circ} \mathrm{C}$ for french marigold (Tagetes patula) 'Janie Flame' to $9.9^{\circ} \mathrm{C}$ for angelonia (Angelonia angustifolia) 'Serena Purple', with petunia 'Dreams Neon Rose'
Plant material and greenhouse culture. Seeds of nine Petunia $\times$ hybrida cultivars (group 1; 'Damask Purple', 'Double Madness Blue', 'Dreams Midnight', 'Duvet Red', 'Madness White', 'Picobella Pink', 'Pretty Grand White', 'Supercascade White', and 'Wave Purple') were sown in 128-cell trays (cell volume, $12 \mathrm{~mL}$ ) in soilless media containing (by volume) $70 \%$ peatmoss, $21 \%$ vermiculite, and 9\% perlite (Suremix; Michigan Grower Products, Galesburg, MI) on 9 Jan. 2017. An additional 11 cultivars (group 2; 'Double Valentine', 'Dreams Burgundy', 'Easy Wave Blue', 'Hulahoop Blue', 'Limbo Red', 'Picobella Cascade White', 'Pirouette Purple', 'Plush Red', 'Prism Sunshine', 'Ramblin' Violet', and 'Tritunia Pink') were sown on 16 Jan. 2017. Seed trays were placed 
in a greenhouse at $23 \pm 1.2{ }^{\circ} \mathrm{C}$ under intermittent mist from reverse-osmosis-treated water under ambient irradiance plus $60 \pm 8$ $\mu \mathrm{mol} \cdot \mathrm{m}^{-2} \cdot \mathrm{s}^{-1}$ supplemental irradiance from high-pressure sodium lamps from 0600 to 2200 HR. Fourteen days after seed sowing, when approximately two true leaves had unfolded, seedlings were transplanted to 10$\mathrm{cm}$-diameter round pots $(450 \mathrm{~mL})$ containing the same soilless media and moved to one of three greenhouse compartments set to a constant temperature of 14,17 , or $20^{\circ} \mathrm{C}$ under a 16-h photoperiod (ambient irradiance plus $90 \pm 17 \mu \mathrm{mol} \cdot \mathrm{m}^{-2} \cdot \mathrm{s}^{-1}$ supplemental irradiance from high-pressure sodium lamps from 0600 to $2200 \mathrm{HR}$ when outdoor irradiance was less than $\left.440 \mu \mathrm{mol} \cdot \mathrm{m}^{-2} \cdot \mathrm{s}^{-1}\right)$. Shielded and aspirated $0.13-\mathrm{mm}$ thermocouples (Type E; Omega Engineering, Stamford, CT) connected to a data logger (CR1000; Campbell Scientific, Logan, UT) recorded the air temperature each minute, and hourly averages were stored. Actual average daily temperatures $(\mathrm{ADTs}) \pm$ SD were $14.7 \pm 1.9,16.8 \pm 1.4$, and $21.0 \pm 1.0^{\circ} \mathrm{C}$ for group 1 and $15.5 \pm 1.7$, $17.3 \pm 1.3$, and $21.3 \pm 1.1{ }^{\circ} \mathrm{C}$ for group 2 . Plants were irrigated as needed with reverseosmosis-treated water supplemented with the following (in $\mathrm{mg} \cdot \mathrm{L}^{-1}$ ): 125 nitrogen, 13 phosphorus, 125 potassium, 15 calcium, 1 iron, 0.5 manganese, 0.5 zinc, 0.5 copper, 0.1 boron, and 0.1 molybdenum (MSU Orchid RO Water Special 13-3-15; GreenCare Fertilizers, Kankakee, IL). There were eight plants per cultivar in each of two completely randomized blocks within each temperature treatment.

Data collection and analysis. When plants were moved to the temperature treatments, the youngest unfolded leaf (leaf length $>1 \mathrm{~cm}$ ) was marked with correction fluid (Liquid Paper; Newell Rubbermaid, Oak Brook, IL). Twenty-one days after the initiation of treatments, the number of new nodes (petunia produces one leaf per node) formed on the primary shoot was determined. This number was then divided by 21 to calculate the development rate (in nodes/d). When the first flower opened on the primary shoot, the date was recorded and the number of nodes below the first flower, number of visible flower buds ( $>3 \mathrm{~mm}$ in length), number of lateral branches $(>5 \mathrm{~cm}$ in length), and plant height (from the media surface to the top of the shoot canopy) were determined. The flowering rate was calculated as $1 /$ time to flower (TTF). The experiment had a split-plot design, with temperature as the main plot and cultivar as the subplot. The block was not significant for any temperature or cultivar; therefore, data were pooled across blocks within a main plot for analysis. Analyses of variance, mean separations [Tukey's honestly significant difference $\left.{ }_{(0.05)}\right]$, Pearson's correlation coefficient determination, and linear regression analyses were conducted using IBM SPSS Statistics v.25 (IBM, Chicago, IL). Linear regression analyses were conducted for the rate of progress toward flowering as a function of temperature calculated using the actual ADT for each temperature treatment, where Rate $=b_{o}+b_{1} \times$ ADT. Base temperature was calculated by setting the rate equal to 0 . Degree-days to flowering was calculated as $1 / b_{1}$.

\section{Results}

For all 20 cultivars, increasing the temperature from 14 to $20{ }^{\circ} \mathrm{C}$ increased the development rate and decreased days to flower (Table 1). However, the relative influence of temperature on TTF varied, resulting in a significant cultivar $\times$ temperature interaction. The reduction in flowering time as temperature increased from 14 to $20{ }^{\circ} \mathrm{C}$ ranged from $17 \mathrm{~d}$ for 'Damask Purple' to $26 \mathrm{~d}$ for 'Wave Purple'. On a relative basis, decreasing the temperature from 20 to $14{ }^{\circ} \mathrm{C}$ increased TTF by $36 \%$ for 'Pirouette Purple' (from 51 to $70 \mathrm{~d}$ ) to $70.3 \%$ for 'Dreams Midnight' (from 37 to $62 \mathrm{~d}$ ), with an average increase of $57.2 \%$ across all cultivars.

Temperature sensitivity of the rate of progress toward flowering (1/TTF) was evaluated by a linear regression analysis (Table 2 ). The variation in cultivar flowering time response to temperature across cultivars was reflected in the variation in the temperature response coefficient of the linear function (i.e., the increase in $1 / \mathrm{TTF}$ per $1{ }^{\circ} \mathrm{C}$ temperature increase). The temperature response coefficient (slope of the linear function) across cultivars averaged 0.00163 and ranged from 0.00096 for 'Double Valentine' to 0.0021 for 'Picobella Pink', indicating that the flowering time of 'Picobella Pink' was more than twice as sensitive to temperature as 'Double Valentine'. The base temperature for flowering (the temperature at which the rate of progress toward flowering is 0 ) averaged $4.3^{\circ} \mathrm{C}$ across all cultivars, ranging from $0.15^{\circ} \mathrm{C}$ for 'Damask Purple' to $7.1{ }^{\circ} \mathrm{C}$ for 'Wave Purple'. Degree-days to flower ranged from 476 for 'Picobella Pink' to 1042 for 'Double Valentine' (Table 2).

Cultivars varied in the number of nodes produced below the first open flower (Table 1). Although temperature did not influence the number of nodes that formed below the first flower for 17 of the cultivars, there was a significant cultivar $\times$ temperature interaction. Two cultivars, 'Limbo Red' and 'Picobella Cascade White', produced fewer nodes below the first flower at $17^{\circ} \mathrm{C}$, compared with 14 or $20^{\circ} \mathrm{C}$. In contrast, 'Duvet Red' produced fewer nodes below the first flower at $14^{\circ} \mathrm{C}$, compared with 17 or $20^{\circ} \mathrm{C}$.

Temperature and cultivar interacted to impact flower bud number at first flowering. The flower bud number of nine of the 20 cultivars was similar across temperatures (Table 1). For the other 11 cultivars, the flower bud number was greater at $14{ }^{\circ} \mathrm{C}$ than at $20^{\circ} \mathrm{C}$. Temperature had the largest impact on the flower bud number of petunia 'Wave Purple', with plants grown at 14 and $17{ }^{\circ} \mathrm{C}$ producing more than double the number of flower buds (68 and 72 buds, respectively) compared to plants grown at $20^{\circ} \mathrm{C}$ ( 32 buds).

Plant height at flowering and branch number were both impacted by cultivar $\times$ tem- perature. The height of 13 cultivars was similar across all temperatures (Table 1). The height of 'Dreams Burgundy', 'Hulahoop Blue', 'Picobella Cascade White', 'Plush Red', and 'Prism Sunshine' decreased as the temperature increased from 14 to $20{ }^{\circ} \mathrm{C}$, whereas the height of 'Double Valentine' and 'Madness White' was lowest at $17{ }^{\circ} \mathrm{C}$. The branch number of 11 cultivars was not impacted by temperature. For six of the other nine cultivars, the branch number was lower at $20{ }^{\circ} \mathrm{C}$ than at 14 or $17{ }^{\circ} \mathrm{C}$. 'Damask Purple', 'Double Madness Blue', and 'Picobella Cascade White' produced more branches at 17 than at $20^{\circ} \mathrm{C}$.

The TTF was negatively correlated with the development rate both within and across temperatures (Table 3), whereas the node number below the first flower was not correlated with TTF in any treatment and marginally correlated positively with the development rate at $14{ }^{\circ} \mathrm{C}$ only $(P=0.026)$. Trait correlations were generally similar when compared either within a temperature treatment or across temperatures, with some exceptions. For example, across all temperatures, there was a strong positive correlation $(P<0.001)$ between TTF and the number of flower buds produced. However, this was largely due to a strong positive correlation at $20{ }^{\circ} \mathrm{C}$ because these traits were not correlated at $17{ }^{\circ} \mathrm{C}$ and only marginally $(P=$ $0.026)$ correlated positively at $14{ }^{\circ} \mathrm{C}$ (Table 3).

\section{Discussion}

Controlling and accurately scheduling flowering time is critical for annual bedding plant production. The TTF is primarily a function of ADT when plants are grown under otherwise appropriate conditions (e.g., proper photoperiod) for flowering. A panel of 20 seed-propagated petunia cultivars grown under a range of temperatures revealed variations in temperature responsiveness for the development and flowering rates, as well as several crop quality parameters. Increasing the temperature from 14 to $20{ }^{\circ} \mathrm{C}$ increased development and flowering rates for all cultivars, but the relative rate change in response to temperature varied widely across cultivars.

A previous study evaluated the temperature sensitivity of flowering time in four Petunia spp. (Warner, 2010). The temperature response coefficients for the rate of progress toward flowering for the species ranged from 0.0010 for $P$. exserta and petunia to 0.0015 for $P$. integrifolia, which was narrower than the range of 0.00096 to 0.0021 exhibited by the cultivars in this study (Table 2), indicating that petunia cultivars represent a wider range of thermal sensitivity for flowering time compared with the smaller group of species, particularly at the higher end (i.e., more temperature-sensitive) of the response spectrum. These temperature response coefficients are within the range of values previously reported for annual bedding plants. For example, linear models were 
Table 1. Effect of temperature on time to flower, development rate (Dev. rate), node number below the first flower (Nodes), plant height at flowering (Height), number of lateral branches (Branches), and number of flower buds at first flowering (Flower buds) of 20 petunia (Petunia $\times$ hybrida) cultivars.

\begin{tabular}{|c|c|c|c|c|c|c|c|}
\hline Cultivar & $\begin{array}{c}\text { Temp } \\
\left({ }^{\circ} \mathrm{C}\right)\end{array}$ & $\begin{array}{l}\text { Dev. rate } \\
\text { (nodes/d) }\end{array}$ & $\begin{array}{l}\text { Time to } \\
\text { flower (d) }\end{array}$ & $\begin{array}{c}\text { Nodes } \\
\text { (no.) }\end{array}$ & $\begin{array}{c}\text { Flower } \\
\text { buds (no.) }\end{array}$ & $\begin{array}{l}\text { Height } \\
(\mathrm{cm})\end{array}$ & $\begin{array}{c}\text { Branches } \\
\text { (no.) }\end{array}$ \\
\hline \multirow[t]{3}{*}{ Damask Purple } & 14 & $0.310 \mathrm{a}^{\mathrm{z}}$ & $52 \mathrm{c}$ & $10.2 \mathrm{a}$ & $26 \mathrm{a}$ & $7.97 \mathrm{a}$ & $8.3 \mathrm{ab}$ \\
\hline & 17 & $0.411 \mathrm{a}$ & $41 \mathrm{~b}$ & $10.5 \mathrm{a}$ & $35 \mathrm{a}$ & $8.88 \mathrm{a}$ & $8.9 \mathrm{~b}$ \\
\hline & 20 & $0.530 \mathrm{~b}$ & $36 \mathrm{a}$ & $11.7 \mathrm{a}$ & $31 \mathrm{a}$ & $8.70 \mathrm{a}$ & $6.9 \mathrm{a}$ \\
\hline \multirow[t]{3}{*}{ Double Madness Blue } & 14 & $0.196 \mathrm{a}$ & $67 \mathrm{c}$ & $11.5 \mathrm{a}$ & $41 \mathrm{a}$ & $11.81 \mathrm{a}$ & $9.4 \mathrm{ab}$ \\
\hline & 17 & $0.292 \mathrm{~b}$ & $51 \mathrm{~b}$ & $12.3 \mathrm{a}$ & $54 \mathrm{a}$ & $9.90 \mathrm{a}$ & $11.0 \mathrm{~b}$ \\
\hline & 20 & $0.388 \mathrm{c}$ & $42 \mathrm{a}$ & $14.0 \mathrm{a}$ & $37 \mathrm{a}$ & $8.61 \mathrm{a}$ & $7.6 \mathrm{a}$ \\
\hline \multirow[t]{3}{*}{ Double Valentine } & 14 & $0.190 \mathrm{a}$ & $73 \mathrm{c}$ & $13.4 \mathrm{a}$ & $45 \mathrm{a}$ & $16.55 \mathrm{~b}$ & $9.8 \mathrm{a}$ \\
\hline & 17 & $0.245 \mathrm{a}$ & $67 \mathrm{~b}$ & $11.3 \mathrm{a}$ & $49 \mathrm{a}$ & $12.69 \mathrm{a}$ & $11.0 \mathrm{a}$ \\
\hline & 20 & $0.363 \mathrm{~b}$ & $53 \mathrm{a}$ & $12.6 \mathrm{a}$ & $41 \mathrm{a}$ & $19.49 \mathrm{~b}$ & $11.0 \mathrm{a}$ \\
\hline \multirow[t]{3}{*}{ Dreams Burgundy } & 14 & $0.214 \mathrm{a}$ & $61 \mathrm{c}$ & $9.5 \mathrm{a}$ & $29 \mathrm{c}$ & $10.74 \mathrm{a}$ & $7.5 \mathrm{a}$ \\
\hline & 17 & $0.268 \mathrm{a}$ & $52 \mathrm{~b}$ & $10.8 \mathrm{a}$ & $24 \mathrm{~b}$ & $10.05 \mathrm{a}$ & $7.4 \mathrm{a}$ \\
\hline & 20 & $0.375 \mathrm{~b}$ & $38 \mathrm{a}$ & $9.5 \mathrm{a}$ & $17 \mathrm{a}$ & $8.91 \mathrm{a}$ & $6.4 \mathrm{a}$ \\
\hline \multirow[t]{3}{*}{ Dreams Midnight } & 14 & $0.183 \mathrm{a}$ & $62 \mathrm{c}$ & $12.5 \mathrm{a}$ & $35 \mathrm{~b}$ & $11.39 \mathrm{~b}$ & $10.8 \mathrm{~b}$ \\
\hline & 17 & $0.253 \mathrm{a}$ & $51 \mathrm{~b}$ & $12.6 \mathrm{a}$ & $30 \mathrm{ab}$ & $10.17 \mathrm{ab}$ & $10.8 \mathrm{~b}$ \\
\hline & 20 & $0.419 \mathrm{~b}$ & $37 \mathrm{a}$ & $12.8 \mathrm{a}$ & $21 \mathrm{a}$ & $8.46 \mathrm{a}$ & $8.0 \mathrm{a}$ \\
\hline \multirow[t]{3}{*}{ Duvet Red } & 14 & $0.179 \mathrm{a}$ & $58 \mathrm{c}$ & $7.7 \mathrm{a}$ & $21 \mathrm{a}$ & $7.53 \mathrm{a}$ & $6.7 \mathrm{a}$ \\
\hline & 17 & $0.256 \mathrm{~b}$ & $45 \mathrm{~b}$ & $11.1 \mathrm{~b}$ & $18 \mathrm{a}$ & $5.58 \mathrm{a}$ & $8.4 \mathrm{a}$ \\
\hline & 20 & $0.423 \mathrm{c}$ & $37 \mathrm{a}$ & $10.4 \mathrm{~b}$ & $15 \mathrm{a}$ & $5.96 \mathrm{a}$ & $7.5 \mathrm{a}$ \\
\hline \multirow[t]{3}{*}{ Easy Wave Blue } & 14 & $0.315 \mathrm{a}$ & $56 \mathrm{c}$ & $13.1 \mathrm{a}$ & $52 \mathrm{a}$ & $7.13 \mathrm{a}$ & $11.4 \mathrm{a}$ \\
\hline & 17 & $0.375 \mathrm{a}$ & $50 \mathrm{~b}$ & $13.4 \mathrm{a}$ & $41 \mathrm{a}$ & $6.30 \mathrm{a}$ & $9.3 \mathrm{a}$ \\
\hline & 20 & $0.494 \mathrm{~b}$ & $37 \mathrm{a}$ & $13.5 \mathrm{a}$ & $37 \mathrm{a}$ & $7.13 \mathrm{a}$ & $10.6 \mathrm{a}$ \\
\hline \multirow[t]{3}{*}{ Hulahoop Blue } & 14 & $0.155 \mathrm{a}$ & $66 \mathrm{c}$ & $9.9 \mathrm{a}$ & $29 \mathrm{~b}$ & $9.74 \mathrm{~b}$ & $10.1 \mathrm{a}$ \\
\hline & 17 & $0.220 \mathrm{~b}$ & $56 \mathrm{~b}$ & $9.8 \mathrm{a}$ & $28 \mathrm{~b}$ & $9.63 \mathrm{~b}$ & $7.8 \mathrm{a}$ \\
\hline & 20 & $0.357 \mathrm{c}$ & $40 \mathrm{a}$ & $8.7 \mathrm{a}$ & $16 \mathrm{a}$ & $7.13 \mathrm{a}$ & $7.6 \mathrm{a}$ \\
\hline \multirow[t]{3}{*}{ Limbo Red } & 14 & $0.250 \mathrm{a}$ & $60 \mathrm{c}$ & $12.0 \mathrm{~b}$ & $31 \mathrm{~b}$ & $8.19 \mathrm{a}$ & $10.1 \mathrm{~b}$ \\
\hline & 17 & $0.298 \mathrm{a}$ & $53 \mathrm{~b}$ & $8.1 \mathrm{a}$ & $24 \mathrm{ab}$ & $8.40 \mathrm{a}$ & $9.0 \mathrm{~b}$ \\
\hline & 20 & $0.417 \mathrm{~b}$ & $37 \mathrm{a}$ & $9.9 \mathrm{ab}$ & $21 \mathrm{a}$ & $6.48 \mathrm{a}$ & $7.0 \mathrm{a}$ \\
\hline \multirow[t]{3}{*}{ Madness White } & 14 & $0.256 \mathrm{a}$ & $58 \mathrm{c}$ & $12.4 \mathrm{a}$ & $32 \mathrm{a}$ & $8.59 \mathrm{~b}$ & $8.7 \mathrm{a}$ \\
\hline & 17 & $0.361 \mathrm{~b}$ & $46 \mathrm{~b}$ & $15.1 \mathrm{a}$ & $35 \mathrm{a}$ & $6.84 \mathrm{a}$ & $9.9 \mathrm{a}$ \\
\hline & 20 & $0.440 \mathrm{c}$ & $38 \mathrm{a}$ & $12.8 \mathrm{a}$ & $25 \mathrm{a}$ & $7.59 \mathrm{ab}$ & $9.4 \mathrm{a}$ \\
\hline \multirow[t]{3}{*}{ Picobella Cascade White } & 14 & $0.327 \mathrm{a}$ & $58 \mathrm{c}$ & $15.1 \mathrm{~b}$ & $50 \mathrm{ab}$ & $8.64 \mathrm{~b}$ & $13.9 \mathrm{ab}$ \\
\hline & 17 & $0.393 \mathrm{a}$ & $50 \mathrm{~b}$ & $9.6 \mathrm{a}$ & $54 \mathrm{~b}$ & $7.34 \mathrm{~b}$ & $16.0 \mathrm{~b}$ \\
\hline & 20 & $0.589 \mathrm{~b}$ & $37 \mathrm{a}$ & $15.6 \mathrm{~b}$ & $34 \mathrm{a}$ & $5.26 \mathrm{a}$ & $11.5 \mathrm{a}$ \\
\hline \multirow[t]{3}{*}{ Picobella Pink } & 14 & $0.262 \mathrm{a}$ & $54 \mathrm{c}$ & $10.6 \mathrm{a}$ & $30 \mathrm{ab}$ & $8.14 \mathrm{a}$ & $11.8 \mathrm{a}$ \\
\hline & 17 & $0.390 \mathrm{~b}$ & $41 \mathrm{~b}$ & $13.4 \mathrm{a}$ & $44 \mathrm{~b}$ & $5.14 \mathrm{a}$ & $11.2 \mathrm{a}$ \\
\hline & 20 & $0.571 \mathrm{c}$ & $33 a$ & $12.2 \mathrm{a}$ & $22 \mathrm{a}$ & $5.35 \mathrm{a}$ & $9.3 \mathrm{a}$ \\
\hline \multirow[t]{3}{*}{ Pirouette Purple } & 14 & $0.214 \mathrm{a}$ & $70 \mathrm{c}$ & $12.5 \mathrm{a}$ & $39 \mathrm{~b}$ & $14.13 \mathrm{a}$ & $10.6 \mathrm{~b}$ \\
\hline & 17 & $0.232 \mathrm{a}$ & $63 \mathrm{~b}$ & $12.8 \mathrm{a}$ & $43 \mathrm{~b}$ & $12.71 \mathrm{a}$ & $10.0 \mathrm{~b}$ \\
\hline & 20 & $0.327 \mathrm{~b}$ & $51 \mathrm{a}$ & $14.0 \mathrm{a}$ & $29 \mathrm{a}$ & $14.06 \mathrm{a}$ & $6.9 \mathrm{a}$ \\
\hline Plush Red & 14 & $0.185 \mathrm{a}$ & $62 \mathrm{c}$ & $11.5 \mathrm{a}$ & $35 \mathrm{~b}$ & $10.95 \mathrm{~b}$ & $10.4 \mathrm{a}$ \\
\hline & 17 & $0.226 \mathrm{a}$ & $55 \mathrm{~b}$ & $9.5 \mathrm{a}$ & $36 \mathrm{~b}$ & $9.58 \mathrm{ab}$ & $9.3 \mathrm{a}$ \\
\hline & 20 & $0.369 \mathrm{~b}$ & $39 a$ & $11.7 \mathrm{a}$ & $21 \mathrm{a}$ & $8.35 \mathrm{a}$ & $8.8 \mathrm{a}$ \\
\hline Pretty Grand White & 14 & $0.229 \mathrm{a}$ & $57 \mathrm{c}$ & $11.4 \mathrm{a}$ & $28 \mathrm{a}$ & $6.98 \mathrm{a}$ & $11.8 \mathrm{~b}$ \\
\hline & 17 & $0.270 \mathrm{a}$ & $47 \mathrm{~b}$ & $13.0 \mathrm{a}$ & $28 \mathrm{a}$ & $6.33 \mathrm{a}$ & $12.3 \mathrm{~b}$ \\
\hline & 20 & $0.503 \mathrm{~b}$ & $35 \mathrm{a}$ & $10.4 \mathrm{a}$ & $20 \mathrm{a}$ & $6.83 \mathrm{a}$ & $8.4 \mathrm{a}$ \\
\hline Prism Sunshine & 14 & $0.226 \mathrm{a}$ & $61 \mathrm{c}$ & $11.9 \mathrm{a}$ & $31 \mathrm{a}$ & $13.79 \mathrm{~b}$ & $8.6 \mathrm{a}$ \\
\hline & 17 & $0.292 \mathrm{a}$ & $54 \mathrm{~b}$ & $11.0 \mathrm{a}$ & $30 \mathrm{a}$ & $11.39 \mathrm{~b}$ & $8.3 \mathrm{a}$ \\
\hline & 20 & $0.417 \mathrm{~b}$ & $38 \mathrm{a}$ & $11.6 \mathrm{a}$ & $26 \mathrm{a}$ & $8.47 \mathrm{a}$ & $8.6 \mathrm{a}$ \\
\hline Ramblin' Violet & 14 & $0.224 \mathrm{a}$ & $59 \mathrm{c}$ & $11.1 \mathrm{a}$ & $37 \mathrm{~b}$ & $7.97 \mathrm{a}$ & $11.7 \mathrm{~b}$ \\
\hline & 17 & $0.279 \mathrm{a}$ & $52 \mathrm{~b}$ & $11.6 \mathrm{a}$ & $29 \mathrm{ab}$ & $7.96 \mathrm{a}$ & $9.6 \mathrm{~b}$ \\
\hline & 20 & $0.405 \mathrm{~b}$ & $38 \mathrm{a}$ & $11.6 \mathrm{a}$ & $23 \mathrm{a}$ & $7.00 \mathrm{a}$ & $6.7 \mathrm{a}$ \\
\hline Supercascade White & 14 & $0.238 \mathrm{a}$ & $59 \mathrm{~b}$ & $11.6 \mathrm{a}$ & $36 \mathrm{a}$ & $9.89 \mathrm{a}$ & $9.8 \mathrm{~b}$ \\
\hline & 17 & $0.262 \mathrm{a}$ & $53 \mathrm{~b}$ & $13.7 \mathrm{a}$ & $34 \mathrm{a}$ & $8.97 \mathrm{a}$ & $9.4 \mathrm{~b}$ \\
\hline & 20 & $0.440 \mathrm{~b}$ & $38 \mathrm{a}$ & $11.9 \mathrm{a}$ & $28 \mathrm{a}$ & $10.54 \mathrm{a}$ & $6.6 \mathrm{a}$ \\
\hline Tritunia Pink & 14 & $0.196 \mathrm{a}$ & $60 \mathrm{c}$ & $9.9 \mathrm{a}$ & $35 \mathrm{~b}$ & $9.16 \mathrm{a}$ & $10.3 \mathrm{a}$ \\
\hline & 17 & $0.256 \mathrm{a}$ & $52 \mathrm{~b}$ & $9.3 \mathrm{a}$ & $32 \mathrm{ab}$ & $8.09 \mathrm{a}$ & $9.4 \mathrm{a}$ \\
\hline & 20 & $0.399 \mathrm{~b}$ & $38 \mathrm{a}$ & $11.0 \mathrm{a}$ & $23 \mathrm{a}$ & $8.03 \mathrm{a}$ & $9.3 \mathrm{a}$ \\
\hline Wave Purple & 14 & $0.252 \mathrm{a}$ & $62 \mathrm{c}$ & $13.6 \mathrm{a}$ & $68 \mathrm{~b}$ & $7.73 \mathrm{a}$ & $11.6 \mathrm{a}$ \\
\hline & 17 & $0.361 \mathrm{~b}$ & $45 \mathrm{~b}$ & $12.2 \mathrm{a}$ & $72 \mathrm{~b}$ & $7.32 \mathrm{a}$ & $11.2 \mathrm{a}$ \\
\hline & 20 & $0.531 \mathrm{c}$ & $36 \mathrm{a}$ & $9.3 \mathrm{a}$ & $32 \mathrm{a}$ & $6.06 \mathrm{a}$ & $9.9 \mathrm{a}$ \\
\hline Cultivar & & $* * *$ & $* * *$ & $* *$ & $* * *$ & $* * *$ & $* * *$ \\
\hline Temperature & & $* * *$ & $* * *$ & NS & $* * *$ & $* * *$ & $* * *$ \\
\hline Temperature $\times$ Cultivar & & NS & * & $* *$ & $* *$ & $* * *$ & $* *$ \\
\hline
\end{tabular}

used to describe the effects of temperature (between 14 and $26^{\circ} \mathrm{C}$ ) on 14 annual bedding plant species (Vaid and Runkle, 2013), with temperature response coefficients ranging from 0.00088 for twinspur (Diascia barbarae) 'Diamonte Mix' to 0.0027 for torenia 'Clown Blue'. In that study, the temperature response coefficient for petunia 'Bravo Blue' was reported as 0.0019 and 0.0026 for spring and fall replications, respectively (Vaid and Runkle, 2013). The difference could be, at least partially, attributed to differences in the 
Table 2. Linear regression equations for the rate of progress toward flowering (1/TTF) as a function of average daily temperature (ADT), calculated base temperature $\left(\mathrm{T}_{\text {base }}\right)$, and degree-days to flowering for 20 petunia (Petunia $\times$ hybrida) cultivars.

\begin{tabular}{|c|c|c|c|c|}
\hline Cultivar & Flowering rate equation & $R^{2}$ & $\begin{array}{l}\text { Flowering } \\
\mathrm{T}_{\text {base }}\left({ }^{\circ} \mathrm{C}\right)\end{array}$ & $\begin{array}{c}\text { Degree-days } \\
\text { to flower }\end{array}$ \\
\hline Double Madness Blue & $1 / \mathrm{TTF}=-0.00330+0.00129 * \mathrm{ADT}$ & 0.789 & 2.5 & 775 \\
\hline Double Valentine & $1 / \mathrm{TTF}=-0.00143+0.00096 * \mathrm{ADT}$ & 0.831 & 1.5 & 1042 \\
\hline Dreams Midnight & $1 / \mathrm{TTF}=-0.01153+0.00181 * \mathrm{ADT}$ & 0.803 & 6.4 & 552 \\
\hline Duvet Red & $1 / \mathrm{TTF}=-0.0049+0.00156 * \mathrm{ADT}$ & 0.826 & 0.31 & 641 \\
\hline Easy Wave Blue & $1 / \mathrm{TTF}=-0.00512+0.00153 * \mathrm{ADT}$ & 0.910 & 3.3 & 654 \\
\hline Madness White & $1 / \mathrm{TTF}=-0.00810+0.00167 * \mathrm{ADT}$ & 0.751 & 4.9 & 599 \\
\hline Picobella Cascade White & $1 / \mathrm{TTF}=-0.00659+0.00160 * \mathrm{ADT}$ & 0.910 & 4.1 & 625 \\
\hline Picobella Pink & $1 / \mathrm{TTF}=-0.01308+0.00210 * \mathrm{ADT}$ & 0.739 & 6.2 & 476 \\
\hline Pirouette Purple & $1 / \mathrm{TTF}=0.002+0.001 * \mathrm{ADT}$ & 0.755 & 2.0 & 1000 \\
\hline Plush Red & $1 / \mathrm{TTF}=-0.01176+0.00177 * \mathrm{ADT}$ & 0.871 & 6.6 & 565 \\
\hline Pretty Grand White & $1 / \mathrm{TTF}=-0.01162+0.00194 * \mathrm{ADT}$ & 0.815 & 6.0 & 515 \\
\hline Prism Sunshine & $1 / \mathrm{TTF}=-0.0070+0.00155 * \mathrm{ADT}$ & 0.951 & 0.45 & 645 \\
\hline
\end{tabular}

Table 3. Pearson correlation coefficients for time to flower (TTF), development rate (Dev. rate), node number below the first flower (Nodes), plant height at flowering (Height), number of lateral branches (Branches), and number of flower buds at first flowering (Flower buds) at three growing temperatures (14, 17, and $20^{\circ} \mathrm{C}$ ) or pooled across temperatures (All).

\begin{tabular}{|c|c|c|c|c|c|c|}
\hline Temperature & Trait & Dev. rate & Nodes & Height & Branches & Flower buds \\
\hline \multirow[t]{3}{*}{$\overline{\text { All }}$} & TTF & $-0.818^{* *}$ & -0.024 & $0.509^{* *}$ & $0.223^{* *}$ & $0.359^{* *}$ \\
\hline & Nodes & & & -0.004 & $0.094 *$ & 0.080 \\
\hline & Height & & & & 0.050 & $0.167 * *$ \\
\hline \multirow[t]{3}{*}{$14{ }^{\circ} \mathrm{C}$} & TTF & $-0.565^{* *}$ & 0.121 & $0.609^{* *}$ & 0.006 & $0.179 *$ \\
\hline & Dev. rate & & $0.184^{*}$ & $-0.318^{* *}$ & 0.122 & $0.177^{*}$ \\
\hline & Nodes & & & 0.102 & $0.332 * *$ & $0.338^{* *}$ \\
\hline \multirow[t]{5}{*}{$17^{\circ} \mathrm{C}$} & TTF & $-0.629 * *$ & -0.052 & $0.517 * *$ & -0.095 & 0.046 \\
\hline & Dev. rate & & 0.014 & $-0.315^{* *}$ & $0.273 * *$ & $0.207^{*}$ \\
\hline & Nodes & & & -0.036 & 0.108 & -0.003 \\
\hline & Height & & & & -0.021 & 0.084 \\
\hline & Branches & & & & & $0.515^{* *}$ \\
\hline \multirow[t]{2}{*}{$20^{\circ} \mathrm{C}$} & TTF & $-0.671 * *$ & 0.061 & $0.709^{* *}$ & 0.118 & $0.432 * *$ \\
\hline & Nodes & & 0.059 & $-0.422 * *$ & $0.194 *$ & -0.037 \\
\hline
\end{tabular}

***Statistical significance at $P \leq 0.05$ or 0.001 , respectively.

daily light integral (DLI) between the replications, with DLI reported as 18 and 9 $\mathrm{mol} \cdot \mathrm{m}^{-2} \cdot \mathrm{d}^{-1}$ for the spring and fall replications, respectively. The higher DLI in spring likely increased the apical meristem temperature relative to air temperature by altering plant energy balance, particularly in the lower air temperature treatments (Faust and Heins, 1998). Because the apical meristem temperature is the primary driver of the development rate (Faust and Heins, 1998; Karlsson et al., 1989), but because the functions are calculated based on air temperature, this could potentially result in a reduced slope for the linear function (i.e., a smaller temperature response coefficient) for the replication with the higher DLI.

Cultivars in this study exhibited a $\mathrm{T}_{\text {base }}$ for flowering that ranged from $0.15^{\circ} \mathrm{C}$ for 'Damask Purple' to $7.1{ }^{\circ} \mathrm{C}$ for 'Wave Purple'. Previously, Blanchard and Runkle (2011) estimated the $T_{\text {base }}$ for flowering of petunia 'Dreams Neon Rose' and 'Wave Purple' as 2.8 and $5.5^{\circ} \mathrm{C}$, respectively, using a temperature range of 5 to $30^{\circ} \mathrm{C}$. The differences in 'Wave Purple' $\mathrm{T}_{\text {base }}$ may be partly attributable to the age of the starting material; for example, the plants used by Blanchard and Runkle (2011) had 7.7 nodes when the experiment began, whereas plants in the current study had two nodes. It is important to note that a low $\mathrm{T}_{\text {base }}$ for a developmental process such as the flowering rate is not necessarily a good predictor of general plant cold tolerance (Vaid and Runkle, 2013). For example, although $P$. exserta exhibited a lower $\mathrm{T}_{\text {base }}$ for the flowering rate than $P$. axillaris, $P$. integrifolia, or petunia 'Mitchell' (Warner, 2010), it was also the most freezing-sensitive of the four species following cold acclimation treatment (Walworth and Warner, 2009).
Temperature only impacted the node number below the first flower for three of the 20 petunia cultivars in this study (Table 1), and the effect was inconsistent. The petunia 'Duvet Red' node number was lower at $14{ }^{\circ} \mathrm{C}$ than at 17 or $20{ }^{\circ} \mathrm{C}$, whereas the 'Limbo Red' node number was higher at $14{ }^{\circ} \mathrm{C}$ compared with $17{ }^{\circ} \mathrm{C}$; the 'Picobella Cascade White' node number was lowest at $17^{\circ} \mathrm{C}$. In a study of 17 bedding plant species, the node number below the first flower decreased as temperature decreased for 12 species (Blanchard and Runkle, 2011), whereas the node number for petunia 'Purple Wave' increased from 18.5 to 23 nodes as the temperature decreased from 25 to $10^{\circ} \mathrm{C}$, and the node number for petunia 'Dreams Neon Rose' was unaffected by this temperature range. Petunia axillaris and $P$. integrifolia are the progenitor species of the cultivated petunia (Bombarely et al., 2016). In a 
previous study, increasing the temperature from 14 to $20{ }^{\circ} \mathrm{C}$ decreased the node number below the first flower for both $P$. axillaris (from 29.1 to 20.1 nodes) and $P$. integrifolia (from 25.3 to 17.4 nodes) (Warner, 2010), but it did not impact the node number of petunia 'Mitchell'. Mattson and Erwin (2003) determined that increasing the temperature from 12 to $24^{\circ} \mathrm{C}$ decreased the node number below the first flower for petunia 'Wave Purple', 'Dreams Rose', and 'Avalanche Pink'. In the narrower temperature range evaluated here, the 'Wave Purple' mean node number below the first flower was not significantly different across temperatures, although the mean did decrease as the temperature increased. The lack of impact of the temperature on node number below the first flower may be partially due to the narrower temperature range examined here compared to previous studies.

The flower bud number is a critical quality trait for annual bedding plants. The flower bud number at first flowering often decreases as temperature increases (Moccaldi and Runkle, 2007; Park et al., 1998; Pramuk and Runkle, 2005; Vaid et al., 2014). Increasing temperature from 14 to $20{ }^{\circ} \mathrm{C}$ in this study decreased the flower bud number of 11 of the 20 cultivars evaluated, with reductions ranging from $32.7 \%$ for 'Picobella Cascade White' to $53.5 \%$ for 'Wave Purple'. Increasing the temperature from 14 to $26^{\circ} \mathrm{C}$ decreased the flower bud number for the petunia progenitor species $P$. axillaris and $P$. integrifolia, but not for petunia 'Mitchell' (Warner, 2010). The variable responses exhibited by the cultivar panel and species indicate that it should be possible to breed for reduced temperature sensitivity of flower bud production.

Temperature impacted the plant height and branch number of less than half of the cultivars in this study. Temperature only impacted the height of seven of the 20 cultivars (Table 1); five cultivars were shorter at $20{ }^{\circ} \mathrm{C}$ than at $14{ }^{\circ} \mathrm{C}$, and two were shortest at $17{ }^{\circ} \mathrm{C}$ (Table 1). Similarly, Blanchard et al. (2011b) reported that the petunia 'Dreams Neon Rose' height at flowering was similar across a temperature range of 14 to $26{ }^{\circ} \mathrm{C}$. The branch number was impacted by temperature for nine of the 20 cultivars. Of these, all produced fewer branches at $20^{\circ} \mathrm{C}$ than at 14 and/or $17{ }^{\circ} \mathrm{C}$. Similar variability in the branching response to temperature has been reported for other petunia genotypes; for example, branch numbers for petunia 'Snow Cloud', 'Dreams Rose', and the petunia progenitor species $P$. axillaris and $P$. integrifolia decreased as the temperature increased from 14 to $20^{\circ} \mathrm{C}$, whereas the petunia 'Mitchell' branch number was not impacted in this temperature range (Kaczperski et al., 1991; Mattson and Erwin, 2003; Warner, 2010).

This study revealed variable temperature sensitivities for developmental processes and crop quality parameters across a panel of 20 petunia cultivars. Understanding how temperature impacts flowering time could allow these petunia cultivars to be grouped into temperature-response groups to im- prove production efficiency. For example, more temperature-sensitive cultivars, such as 'Picobella Pink' and 'Wave Purple', should not be grown at lower temperatures because that would result in a larger increase in production time than that for less temperature-sensitive cultivars. These results also underscore the importance of developing cultivar-specific temperatureresponse models to allow accurate predictions of crop scheduling times and other temperature responses.

\section{Literature Cited}

Adams, S.R., P. Hadley, and S. Pearson. 1998. The effects of temperature, photoperiod, and photosynthetic photon flux on the time to flowering of petunia 'Express Blush Pink'. J. Amer. Soc. Hort. Sci. 123:577-580.

Adams, S.R., S. Pearson, and P. Hadley. 1997. The effects of temperature, photoperiod and light integral on the time to flowering of pansy $\mathrm{cv}$. Universal Violet (Viola $\mathrm{x}$ wittrockiana Gams.). Ann. Bot. 80:107-112.

Adams, S.R., S. Pearson, P. Hadley, and W.M. Patefield. 1999. The effects of temperature and light integral on the phases of photoperiod sensitivity in Petunia xhybrida. Ann. Bot. 83:263-269.

Blanchard, M.G. and E.S. Runkle. 2011. Quantifying the thermal flowering rates of eighteen species of annual bedding plants. Scientia Hort. 128:30-37.

Blanchard, M.G., E.S. Runkle, and P.R. Fisher. 2011a. Modeling plant morphology and development of petunia in response to temperature and photosynthetic daily light integral. Scientia Hort. 129:313-320.

Blanchard, M.G., E.S. Runkle, and J.M. Frantz. 2011b. Energy-efficient greenhouse production of Petunia and Tagetes by manipulation of temperature and photosynthetic daily light integral. Acta Hort. 893:857-864.

Bombarely, A., M. Moser, A. Amrad, M. Bao, L. Bapaume, C.S. Barry, M. Bliek, M.R. Boersma, L. Borghi, R. Bruggmann, M. Bucher, N. D'Agostino, K. Davies, U. Druege, N. Dudareva, M. Egea-Cortines, M. Delledonne, N. Fernandez-Pozo, P. Franken, L. Grandont, J.S. Heslop-Harrison, J. Hintzsche, M. Johns, R. Koes, X. Lv, E. Lyons, D. Malla, E. Martinoia, N.S. Mattson, P. Morel, L.A. Mueller, J. Muhlemann, E. Nouri, V. Passeri, M. Pezzotti, Q. Qi, D. Reinhardt, M. Rich, K.R. Richert-Poggeler, T.P. Robbins, M.C. Schatz, M.E. Schranz, R.C. Schuurink, T. Schwarzacher, K. Spelt, H. Tang, S.L. Urbanus, M. Vandenbussche, K. Vijverberg, G.H. Villarino, R.M. Warner, J. Weiss, Z. Yue, J. Zethof, F. Quattrocchio, T.L. Sims, and C. Kuhlemeier. 2016. Insight into the evolution of the Solanaceae from the parental genomes of Petunia hybrida. Nat. Plants 2:16074.

Clough, E.A., A.C. Cameron, R.D. Heins, and W.H. Carlson. 2001. Growth and development of Oenothera fruiticosa is influenced by vernalization duration, photoperiod, forcing temperature, and plant growth regulators. J. Amer. Soc. Hort. Sci. 126:269-274.

Faust, J.E. and R.D. Heins. 1998. Modelling shoottip temperature in the greenhouse environment. J. Amer. Soc. Hort. Sci. 123:208-214.

Guo, Y., W.K. Lin, Q. Chen, V.A. Vallejo, and R.M. Warner. 2017. Genetic determinants of crop timing and quality traits in two interspecific Petunia recombinant inbred line populations. Sci. Rep. 7:3200.
Kaczperski, M.P., W.H. Carlson, and M.G. Karlsson. 1991. Growth and development of Petunia $\times$ hybrids as a function of temperature and irradiance. J. Amer. Soc. Hort. Sci. 116:232-237.

Kanellos, E.A.G. and S. Pearson. 2000. Environmental regulation of flowering and growth of Cosmos atrosanguineus (Hook.) Voss. Scientia Hort. 83:265-274.

Karlsson, M.G., R.D. Heins, and J.E. Erwin. 1988. Quantifying temperature controlled leaf unfolding rates in Lilium longiflorum Thunb. J. Amer. Soc. Hort. Sci. 113:70-74.

Karlsson, M.G., R.D. Heins, J.E. Erwin, and R.D. Berghage. 1989. Development rate during four phases of chrysanthemum growth as determined by preceding and prevailing temperatures. J. Amer. Soc. Hort. Sci. 114:234-240.

Mattson, N.S. and J.E. Erwin. 2003. Temperature affects flower initiation and development rate of Impatiens, Petunia, and Viola. Acta Hort. 624:191-197.

Moccaldi, L.A. and E.S. Runkle. 2007. Modeling the effects of temperature and photosynthetic daily light integral on growth and flowering of Salvia splendens and Tagetes patula. J. Amer. Soc. Hort. Sci. 132:283-288.

Niu, G., R.D. Heins, A. Cameron, and W. Carlson. 2001. Temperature and daily light integral influence plant quality and flower development of Campanula carpatica 'Blue Clips', 'Deep Blue Clips', and Campanula 'Birch Hybrid'. HortScience 36:664-668

Niu, G., R.D. Heins, A.C. Cameron, and W.H. Carlson. 2000. Day and night temperatures, daily light integral, and $\mathrm{CO}_{2}$ enrichment affect growth and flower development of pansy (Viola $\mathrm{x}$ wittrockiana). J. Amer. Soc. Hort. Sci. 125:436-441.

Park, B.H., N. Oliveira, and S. Pearson. 1998. Temperature affects growth and flowering of the baloon flower [Platycodon grandiflorus (Jacq.) A. DC. cv. Astra Blue]. HortScience 33:233-236.

Pietsch, G.M., W.H. Carlson, R.D. Heins, and J.E. Faust. 1995. The effect of day and night temperature and irradiance on development of Catharanthus roseus (L.) 'Grape Cooler'. J. Amer. Soc. Hort. Sci. 120:877-881.

Pramuk, L.A. and E.S. Runkle. 2005. Modeling growth and development of Celosia and Impatiens in response to temperature and photosynthetic daily light integral. J. Amer. Soc. Hort. Sci. 130:813-818

Tollenaar, M., T.B. Daynard, and R.B. Hunter. 1979. Effect of temperature on rate of leaf appearance and flowering date in maize. Crop Sci. 19:363-366.

Vaid, T.M. and E.S. Runkle. 2013. Developing flowering rate models in response to mean temperature for common annual ornamental crops. Scientia Hort. 161:15-23.

Vaid, T.M., E.S. Runkle, and J.M. Frantz. 2014. Mean daily temperature regulates plant quality attributes of annual ornamental plants. HortScience 49:574-580.

Walworth, A.E. and R.M. Warner. 2009. Differential cold acclimation ability of Petunia spp. HortScience 44:1219-1222.

Warner, R. and J. Erwin. 2005. Prolonged high temperature exposure and daily light integral impact growth and flowering of five herbaceous ornamental species. J. Amer. Soc. Hort. Sci. 130:319-325.

Warner, R. and J. Erwin. 2006. Prolonged hightemperature exposure differentially reduces growth and flowering of 12 Viola $\mathrm{x}$ wittrockiana Gams. cvs. Scientia Hort. 108:295-302. 
Warner, R.M. 2010. Temperature and photoperiod influence flowering and morphology of four Petunia spp. HortScience 45:365-368.

Warner, R.M. and A.E. Walworth. 2010. Quantitative inheritance of crop timing traits in interspecific hybrid Petunia populations and interactions with crop quality parameters. J. Hered. 101:308316.

White, J.W. and I.J. Warrington. 1984. Growth and development responses of geranium to temperature, light integral, $\mathrm{CO}_{2}$, and chlormequat. J. Amer. Soc. Hort. Sci. 109:728-735.
Yuan, M., W.H. Carlson, R.D. Heins, and A.C Cameron. 1998. Effect of forcing temperature on time to flower of Coreopsis grandiflora, Gaillardia $\times$ grandiflora, Leucanthemum $\times$ superbum, and Rudbeckia fulgida. HortScience 33:663667. 